\title{
Geometry of the anisotropic minimal surfaces
}

\author{
Ivaïlo M. Mladenov and Mariana Ts. Hadzhilazova
}

\begin{abstract}
A simple modification of the surface tension in the axisymmetric case leads to analogues of the Delaunay surfaces. Here we have derived an explicit parameterization of the most simple case of this new class of surfaces which can be considered as a generalization of the catenoids. The geometry of these surfaces depends on two real parameters and has been studied in some detail.
\end{abstract}

\section{Introduction}

In aqueous solution, amphiphilic molecules (e.g., phospholipids) form bilayers, the hydrophilic heads of these molecules being located in both outer sides of the bilayer, which are in contact with the liquid, while their hydrophobic tails remain at the interior. The handbook [7] is a good starting point for learning more about this phenomena.

A bilayer may form a closed membrane which bear the name vesicle. Vesicles constitute a well-defined and sufficiently simple model system for studying the basic physical properties of the more complex cell biomembranes which

Key Words: Delaunay surfaces, membranes, general shape equation.

2010 Mathematics Subject Classification: 53A05, 53A10, 53B50.

Received: August, 2011.

Accepted: February, 2012 
differ in form and function. The equilibrium shapes of the lipid vesicles are determined as the extremals of the functional

$$
F[\mathcal{S}]=\frac{k_{c}}{2} \int_{\mathcal{S}}(2 H+\mathbb{h})^{2} \mathrm{~d} A+\bar{k} \int_{\mathcal{S}} K \mathrm{~d} A+\sigma \int_{\mathcal{S}} \mathrm{d} A+p \int_{\mathcal{S}} \mathrm{d} V .
$$

Here $H, K$ - denote the mean, respectively the Gaussian curvatures of the middle surface $\mathcal{S}$ and $\mathrm{d} A, \mathrm{~d} V$ - the infinitesimal area and volume elements. Additionally, in this model the following physical parameters are taken into account

- the bending rigidity and tensile stress of the membrane

- the spontaneous curvature of the bilayer

- the osmotic pressure difference between both sides of the bilayer.

\section{The General Membrane Shape Equation}

The corresponding Euler-Lagrange equation (Ou-Yang \& Helfrich [13])

$$
2 k_{c} \Delta_{S} H+k_{c}(2 H+\mathbb{h})\left(2 H^{2}-\mathbb{h} H-2 \bar{k}\right)-2 \sigma H+p=0
$$

is often referred to as the general membrane shape equation. Here $\Delta_{S}$ is the Laplace-Beltrami operator on the surface $\mathcal{S}, k_{c}$ and $\bar{k}$ - denote the bending and the Gaussian rigidities of the membrane, $\sigma$ - the tensile stress of the membrane, Ih - the spontaneous curvature of the bilayer and $p$ - the osmotic pressure difference between the external and internal part. Its explicit solutions are discussed in $[1,4,5,6,9,10,14,16]$ and in [15] the reader will find a whole chapter devoted to this subject.

From mathematical point of view the main difficulty in solving (2) is that it represents a nonlinear fourth order partial differential equation for the position vector $\mathbf{x}$ running on the surface $\mathcal{S}$. A fortunate circumstance is that this differential equation can be rewritten in the form of a system of four differential equations of second order. One, namely (2) for the mean curvature $H$ and three others, namely

$$
\Delta_{S} \mathbf{x}=2 H \mathbf{n}
$$


for the components of the position vector $\mathbf{x}$. Here $\mathbf{n}$ stands for the unit normal vector of $\mathcal{S}$ and the formal proof of (3) in the case of orthogonal parameterization can be found either in [11] or [15]. The general form of the surface Laplacian and its action on scalar and vector functions on $\mathcal{S}$ is discussed in depth in [8].

\section{Axisymmetric Membranes}

Most of the above mentioned difficulties disappears if we restrict our considerations to the surfaces of revolution. In what follows they will be described by the position vector $\mathbf{x}$ (assuming that the coordinate axis $O Z$ is the symmetry axis) of the form

$$
\mathbf{x}(z, \phi)=(r(z) \cos \phi, r(z) \sin \phi, z), \quad z \in \mathbb{R}, \quad \phi \in[0,2 \pi) .
$$

Let us consider the problem of extremizing the lateral area of such membrane under the constraint of a fixed volume. The respective functional is

$$
F[r(z)]=2 \pi \int_{0}^{z}\left(\sigma r \sqrt{1+\dot{r}^{2}}-\frac{p}{2} r^{2}\right) \mathrm{d} z
$$

in which $\dot{r}$ denotes $\frac{\mathrm{d} r(z)}{\mathrm{d} z}$. The expression under the integral is called a Lagrangian and will be denoted by $L$. Writing down the Euler-Lagrange equation for $L$ one gets the equation which is known as a Laplace-Young law

$$
p=2 \sigma H
$$

In deriving it use has been made of the existence of the first integral

$$
L-\dot{r} \frac{\partial L}{\partial \dot{r}}=\tilde{T}, \quad \tilde{T}=\text { constant }
$$

which exists in the case when the Lagrangian does not depend explicitly on the independent variable $z$. From (6) it is clear also that if $p$ and $\sigma$ are constants then the mean curvature $H$ is also a constant. The class of constant mean curvature surfaces of revolution were introduced and classified by the French geometer Delaunay [3] and nowadays bears his name. Besides the plane and the catenoid which are examples of Delaunay surfaces with zero 


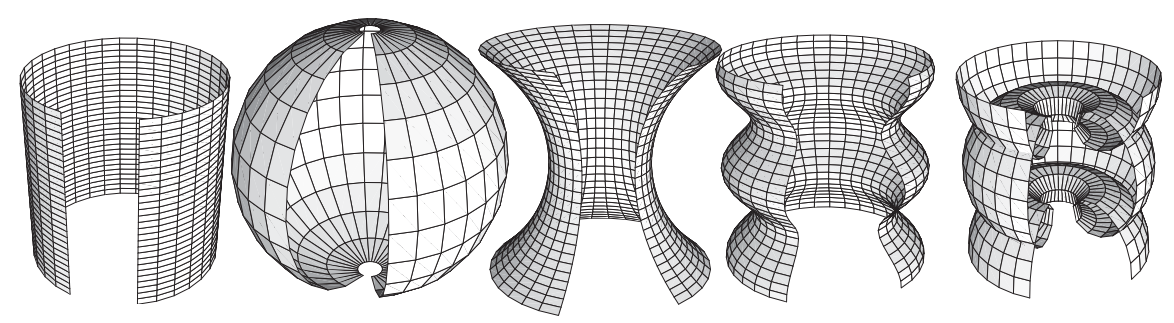

Figure 1: The open parts of the cylinder, sphere, catenoid, unduloid and nodoid.

mean curvatures this class consists of cylinders, spheres, unduloids and nodoids shown in Fig.1.

Actually, Delaunay has proved also that this latter surfaces can be obtained by revolving the traces of the foci of quadrics rolling along the symmetry axis (roulettes in French). The upper part of the Fig.2 show the profile curves (meridional sections of the surfaces) and the lower one is the list of the quadrics generating them.

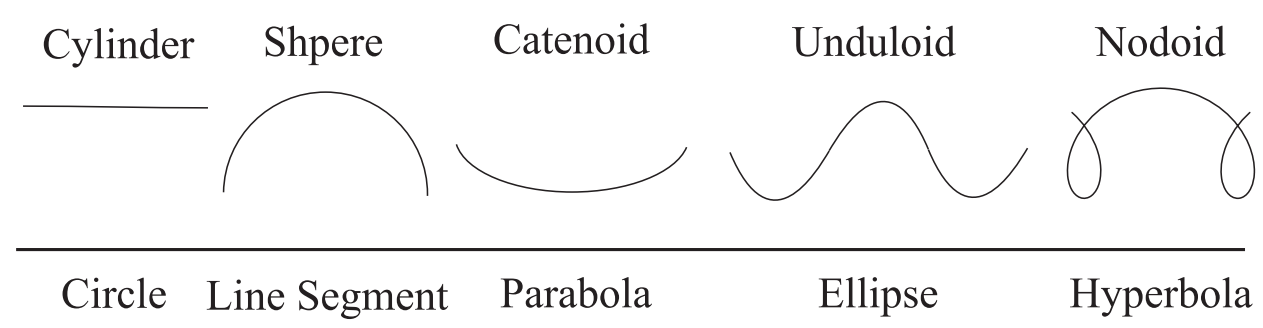

Figure 2: The profile curves and the quadrics generating them.

\section{Modified Delaunay Surfaces}

In this section we will consider (following [2]) a broader class of functionals by abandoning the fundamental up to now assumption $\sigma=$ const about the surface tension and allowing it to depends on the point of the surface. 
In technical terms this means that we have to replace $\sigma$ in (5) with the expression $\sigma+K(r)$ where the function $K(r)$ could be any even function of $r$ (the latter requirement follows from the axial symmetry of the problem), i.e.,

$$
F[r(z)]=2 \pi \int_{0}^{z}\left((\sigma+K(r)) r \sqrt{1+\dot{r}^{2}}-\frac{p}{2} r^{2}\right) \mathrm{d} z, \quad K(-r)=K(r) .
$$

Another possible interpretation is that we are considering a membrane which surface energy is spatially varying. Further on we will analyze the case when $K(r)=\frac{\tilde{\lambda}}{r^{2}}$ which corresponds to the nematic films [2] and respectively we have

$$
\tilde{F}[r(z)]=2 \pi \int_{0}^{z}\left(\left(\sigma+\frac{\tilde{\lambda}}{r^{2}}\right) r \sqrt{1+\dot{r}^{2}}-\frac{p}{2} r^{2}\right) \mathrm{d} z .
$$

The integral of the Euler-Lagrange equation associated with (9) reads

$$
\frac{r}{\sqrt{1+\dot{r}^{2}}}\left(\sigma+\frac{\tilde{\lambda}}{r^{2}}\right)-\frac{p}{2} r^{2}=\tilde{T} .
$$

The general problem presented by (10) will be investigated in the future and here we will treat only the particular case $p=0$ which should be viewed as a study of the generalized catenoids. In this setting it turns out convenient to rescale the free parameters in the model as follows

$$
\lambda=\frac{\tilde{\lambda}}{\sigma}, \quad c=\frac{\tilde{T}}{\sigma}
$$

In this way (10) takes the reduced form

$$
\frac{r}{\sqrt{1+\dot{r}^{2}}}\left(1+\frac{\lambda}{r^{2}}\right)=c
$$

which can be solved with respect to $\dot{r}$ and in this way we end up with the equation

$$
\frac{\mathrm{d} r(z)}{\mathrm{d} z}=\frac{\sqrt{r^{4}(z)+\left(2 \lambda-c^{2}\right) r^{2}(z)+\lambda^{2}}}{c r(z)} .
$$

Introduction of the new variable $\xi=r^{2}$ transforms it into the form

$$
\frac{\mathrm{d} \xi}{\sqrt{\xi^{2}+\left(2 \lambda-c^{2}\right) \xi+\lambda^{2}}}=\frac{2}{c} z
$$


which can be easily integrated and gives

$$
z=\frac{c}{2} \operatorname{arcsinh} \frac{2 \xi+2 \lambda-c^{2}}{c \sqrt{4 \lambda-c^{2}}}
$$

and finally

$$
r(z)=\frac{\sqrt{c \sqrt{4 \lambda-c^{2}} \sinh (2 z / c)+c^{2}-2 \lambda}}{\sqrt{2}} .
$$

The parameterization of the surface $\mathcal{S}$ via $z$ and $\phi$ is not quite suitable for studying the geometry of the generalized catenoids. Instead of $z$ we will introduce a new coordinate $u$ which is related to the old one by the formula

$$
z=\frac{c}{2} \operatorname{arcsinh}(u)
$$

and respectively

$$
r(u)=\frac{\sqrt{c \sqrt{4 \lambda-c^{2}} u+c^{2}-2 \lambda}}{\sqrt{2}} .
$$

The azimuthal coordinate will be denoted as usual in the classical differential geometry textbooks [12] by $v$. In these new coordinates our surface $\mathcal{S}$ is parameterized as follows

$$
\mathbf{x}(u, v)=\left(r(u) \cos v, r(u) \sin v, \frac{c}{2} \operatorname{arcsinh}(u)\right), \quad u \in \mathbb{R}, \quad v \in[0,2 \pi)
$$

and depicted in Fig.3 along its profile curve.

Having an explicit parameterization of any surface one can obtain finer details about it say, various kind of curvatures, geodesic, formulas for the surface area and the volume, etc. For the lack of space we will reproduce here only the formulas for the mean $H$ and Gaussian $K$ curvatures just to see that they are of rational type and therefore easily manageable via analytic and numerical treatment. Introducing $\omega=\sqrt{4 \lambda-c^{2}}$ these formulas are of the form

$H=\frac{4 \lambda(c+\omega u)}{c\left(c^{2}+2 c \omega u+\omega^{2} u^{2}\right)^{3 / 2}}, \quad K=\frac{4\left(4 \lambda c-c^{3}+2 \omega\left(2 \lambda-c^{2}\right) u-c \omega^{2} u^{2}\right)}{c\left(c^{2}+2 c \omega u+\omega^{2} u^{2}\right)^{2}}$ 

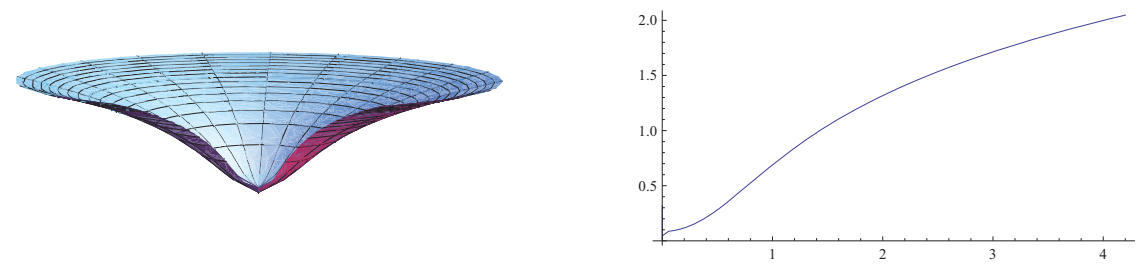

Figure 3: An open part and the corresponding profile curve of $\mathcal{S}$ generated with parameters $\lambda=3 / 5$ and $c=1$.

from which one easily concludes that for a large values of $u$ they both approach the zero, i.e., for such values of the parameter the surface becomes a part of the plane (which can be seen also on the left side of Fig. 3).

A more detailed study of this and the general case (10) is under investigation and will be reported elsewhere.

\section{Acknowledgments}

This research is partially supported by the contract \# 35/2009 between the Bulgarian and Polish Academies of Sciences. The second named author would like to acknowledge the support from the HRD Programme - \# BG051PO0013.3.04/42, financed by the European Union through the European Social Fund.

\section{References}

[1] Blyth M. and Pozrikidis C., Solution Space of Axisymmetric Capsules Enclosed by Elastic Membranes, Eur. J. Mech. A 23 (2004) 877-892.

[2] Chen B. and Kamien R., Nematic Films and Radially Anisotropic Delaunay Surfaces, Eur. Phys. J. E 28 (2009) 315-329.

[3] Delaunay C., Sur la surface de revolution dont la courbure moyenne est constante, J. Math. Pures et Appliquées, 6 (1841) 309-320. 
[4] Djondjorov P., Hadzhilazova M., Mladenov I. and Vassilev V., Beyond Delaunay Surfaces, J. Geom. Symmetry Phys. 18 (2010) 1-11.

[5] Hu J.-G. and Ou-Yang Z.-C., Shape Equations of the Axisymmetric Vesicles, Phys. Rev. E 47 (1993) 461-467.

[6] Konopelchenko B., On Solutions of the Shape Equation for Membranes and Strings, Phys. Lett. B 414 (1997) 58-64.

[7] Lipowsky R. and Sackmann E. (Eds), Handbook of Biological Physics (Structure and Dynamics of Membranes) vol 1, Elsevier, Amsterdam 1995.

[8] Ludu A., Nonlinear Waves and Solitons on Contours and Closed Surfaces, Springer, Berlin 2007.

[9] Mladenov I., New Solutions of the Shape Equation, Eur. Phys. J. B 29 (2002) 327-330.

[10] Mladenov I., Hadzhilazova M., Djondjorov P. and Vassilev V., Some Explicit Solutions of the Shape Equation, In: AIP Conference Proceedings 1301 (2010) 215-227.

[11] Nitsche J., Boundary Value Problems for Variational Integrals Involving Surface Curvatures, Quarterly Appl. Math. 60 (1993) 363-387.

[12] Oprea J., Differential Geometry and Its Applications, Mathematical Association of America, Washington D. C. 2007.

[13] Ou-Yang Z.-C. and Helfrich W., Bending Energy of Vesicle Membranes: General Expressions for the First, Second and Third Variation of the Shape Energy and Applications to Spheres and Cylinders, Phys. Rev. A 39 (1989) 5280-5288.

[14] Ou-Yang Z.-C., Selection of Toroidal Shape of Partially Polymerized Membranes, Phys. Rev. E 47 (1993) 747-749.

[15] Ou-Yang Z.-C., Liu J.-X., and Xie Y.-Z., Geometric Methods in the Elastic Theory of Membranes in Liquid Crystal Phases, World Scientific, Hong Kong 1999. 
[16] Vassilev V., Djondjorov P. and Mladenov I., Cylindrical Equilibrium Shapes of Fluid Membranes, J. Phys. A: Math. \& Theor. 41 (2008) 435201 (16pp); doi:10.1088/1751-8113/41/43/435201.

Ivaïlo M. MLADENOV,

Institute of Biophysics, Bulgarian Academy of Sciences, 1113 Sofia, Bulgaria.

Email: mladenov@obzor.bio21.bas.bg

Mariana Ts. HADZHILAZOVA,

Institute of Biophysics, Bulgarian Academy of Sciences, 1113 Sofia, Bulgaria.

Email: murryh@obzor.bio21.bas.bg 
